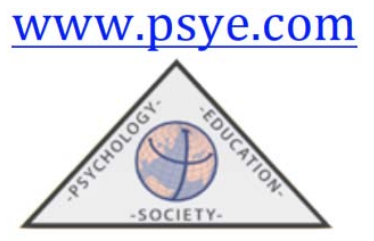

(C) Psychology, Society, \& Education, 2017. Vol. 9(1), pp. 147-161

ISSN 2171-2085 (print) / ISSN 1989-709X (online)

\title{
Impacto de la percepción de los estudiantes respecto de la convivencia escolar sobre su rendimiento académico en Matemáticas
}

\author{
Gamal CERDA ETCHEPARE*; Yasna SALAZAR LLANOS*; Katia SÁEZ \\ CARRILLO*; Carlos PÉREZ WILSON** y José A. CASAS **** \\ * Universidad de Concepción, Chile. **Universidad de O’Higgins, Chile \\ *** Universidad de Córdoba, España.
}

(Recibido 27 Julio, 2016; Aceptado 18 Septiembre, 2016)

RESUMEN: Esta investigación se inscribe dentro de las nuevas líneas de investigación que abordan la influencia de los factores contextuales sobre el rendimiento escolar en matemáticas. En particular, se examina si la percepción que tienen los alumnos acerca de diversas dimensiones de la convivencia escolar tiene incidencia en su logro académico. La muestra de estudio estuvo compuesta por 314 estudiantes chilenos. Los modelos de regresión ordinal y logística, muestran el impacto negativo sobre el rendimiento escolar en matemáticas que tienen las dimensiones de Agresión e Indisciplina del constructo convivencia escolar, especialmente para el grupo femenino que asiste a establecimientos escolares de mayor vulnerabilidad social.

Se discuten las implicaciones y proyecciones de estos resultados para gestión del profesorado de matemáticas al interior del aula escolar, como también, de las autoridades y agentes de políticas educativas.

Palabras clave: Matemáticas, Convivencia escolar, rendimiento, indisciplina, género.

\section{Impact of students' perception of school social environment on their mathematical academic performance}

\begin{abstract}
This study involves a new line of research related to the influence of contextual factors on school performance in mathematics. Specifically, it examines whether the students' perception of different aspects of the school environment has an impact on their academic achievement. The study sample was comprised of 314 Chilean students. Ordinal and logistic regression models revealed a negative impact on school performance in mathematics for the dimensions of Aggression and Indiscipline of the school environment construct, especially for female students who attend schools of high vulnerable social contexts. We discuss the implications and importance of these results in order to improve the math teaching process within the classroom setting, and also to inform authorities who are responsible for establishing educational policies.
\end{abstract}

Keywords: Mathematics, School environment, performance, indiscipline, gender.

${ }^{1}$ Correspondencia: Gamal Cerda, Departmento de Metodología de la Investigación e Informática Educativa. Facultad de Educación, Universidad de Concepción, Chile. gamal.cerda@udec.cl. 
En la actualidad el rendimiento y nivel de logro académico alcanzado por los estudiantes en matemáticas sigue siendo motivo de preocupación, especialmente a la luz de los bajos resultados que ponen de manifiesto las diversas mediciones de carácter internacional (Agencia de Calidad de la Educación, 2014; MINEDUC, 2010a; 2010b, 2014). Las investigaciones realizadas han permitido centrar la mirada en el entorno escolar o directamente sobre el escenario de aprendizaje o clima de aula, que permitan relevar cómo este contexto incide sobre los procesos de activación, adquisición y construcción del conocimiento escolar y su incidencia en la obtención de rendimientos académicos positivos (Arens, Morin, \&Watermann, 2015; Henrich, Schwab-Stone, Fanti, Jones, \&Ruchkin; 2004).

Este enfoque de investigación ha permitido incorporar variables o factores relativos al entorno escolar $y$, en específico, al rol que puede desempeñar el clima escolar sobre el rendimiento académico de los estudiantes. El estudio acerca del impacto positivo o negativo que puede tener en el aprendizaje y logro en matemáticas, el tipo de interacciones, experiencias y percepción que viven los estudiantes al interior de la institución escolar, constituye una línea de investigación abierta y novedosa.

El concepto de convivencia escolar es polisémico, y por lo mismo difícil de definir en forma simple. Sus definiciones más bien dan cuenta de la amplitud de elementos y factores que son referidos a la hora de su análisis (Cohen, McCabe, Michelli, \&Pickeral, 2009; Gázquez, Pérez, \& Carrión, 2011; González-Castro, Álvarez-García, Rodríguez, Álvarez, \& Núñez, 2010). La presente investigación asume una concepción más global, cercana al concepto de "clima escolar" que involucra a todos los agentes de la institución u organización escolar (Sánchez-Moreno, \& López Yáñez, 2010; Zullig, Koopman, Patton, \&Ubbes, 2010). En Chile, la Ley sobre Violencia Escolar promulgada en Chile (Ley 20.352, 2011), y sus diversos artículos expresan con nitidez que la convivencia escolar es un tema relevante, y que ella se funda en la coexistencia armónica y en una interrelación positiva de todos los miembros de la comunidad educativa. La institución escolar es el espacio de interacción social más adecuado para experimentar la convivencia, y además, si ella es positiva y sana, de seguro repercutirá positivamente en el aprendizaje, pues en ella es posible articular un ambiente propicio para éste (Arístegui, Bazán, Leiva, López, Muñoz, \& Ruz, 2005). Si bien la convivencia escolar tiene un impacto directo en los aprendizajes, este impacto puede ser de connotación negativa si se evidencian por ejemplo situaciones de intimidación, las cuales interfieren negativamente (Toledo, Gutiérrez, \&Magendzo, 2009).

Se destaca que un clima positivo en la escuela juega un papel protector en el desarrollo social, emocional y académico el desarrollo de jóvenes (De Pedro, Gilreath, \&Berkowitz, 2016). Del mismo modo, la gestión positiva del docente es muy importante a la hora de generar entornos de aprendizaje estimulantes, y en ese propósito es probable que el logro de aprendizaje se fortalezca más si el estilo de los profesores en su interacción en el aula, se enmarca dentro de un modelo interaccionista más que un modelo intervencionista (Djigic, \&Stojiljkovic, 2011). El nivel de compromiso docente, entendido como el nivel de involucramiento y supervisión en las tareas académicas de los estudiantes por parte de los profesores y las buenas relaciones interpersonales con los padres tienen un efecto positivo en el rendimiento en matemáticas (Kodzi, et al., 2014).

Existe evidencia que muestra que el compromiso de enseñanza por parte de los 
profesores, expresado en acciones específicas, como fomentar la participación en la clase y corregir las tareas de los estudiantes, se relaciona positivamente con un mejor desempeño de éstos en la asignatura de matemáticas. Esta supervisión activa por parte de los profesores, conjuntamente con buenas relaciones interpersonales con los padres, tiene un efecto positivo en dicho rendimiento (Kodzi, et al., 2014). Las percepciones de aula pueden ejercer una influencia indirecta sobre el rendimiento al aumentar el interés y el auto-concepto, factores intrapersonales que a su vez tienen una relación directa con el logro y pueden mediar en la relación entre el ambiente de la clase percibida y la habilidad matemática (Tosto, Asbury, Mazzocco, Petrill, \& Kovas, 2016).

En la relación entre el clima escolar y el rendimiento académico, es especialmente significativo relevar las situaciones de indisciplina que ocurren al interior del aula escolar, pues ellas perturban el logro de objetivos de aprendizaje y en muchos casos la motivación de los estudiantes (Arens, Morin, \&Watermann, 2015). El tipo de gestión del aula es una de las competencias fundamentales del profesor a la hora de configurar ambientes propicios para el aprendizaje. Si los docentes utilizan estrategias de disciplina de exclusión, éstas se asocian con puntuaciones de orden y de disciplina más bajos de sus estudiantes, mientras que un mayor uso de los apoyos de comportamiento positivo en el aula se asocian con puntuaciones más altas en el orden y la disciplina, la equidad, y una mejor valoración de la relación estudiante-profesor (Mitchell, \& Bradshaw, 2013).

Por otra parte, en contextos de alta vulnerabilidad social, las percepciones de los estudiantes acerca del clima de la escuela moderan la relación siempre estrecha entre pobreza y desempeño académico bajo y con tipos de comportamiento inadecuados. Tanto es así, que los estudiantes de familias pobres que perciben un positivo clima escolar favorable exhiben comportamientos similares a sus pares de familias de ingresos más altos (Hopson, \& Lee, 2011). Se ha logrado comprobar que controlando variables relativas a la composición de la escuela, el clima escolar tiene una gran importancia en la explicación de los resultados académicos de los estudiantes, y que éste, a su vez, genera un efecto indirecto sobre las expectativas de los profesores (Brault, Janosz, \&Archambaul, 2014). Existe también evidencia que un clima escolar favorable influye positivamente en las creencias de los profesores noveles o principiantes respecto de su auto-eficacia profesional y, por ende, en su probable eficacia docente (Meristo, \&Eisenschmidt, 2014). Muestras de percepciones de profesores a gran escala acerca del clima de las organizaciones educativas donde trabajan, evidencian que ellas se asocian de manera significativa y consistente con el desempeño de sus estudiantes en las pruebas estandarizadas de rendimiento académico, pero también, con medidas de ajuste académico, comportamiento y socio-emocionales (Brand, Felner, Seitsinger, Burns, \& Bolton, 2008).

Por otra parte, diversas investigaciones demuestran que ser víctima de acoso o intimidación escolar tiene un efecto negativo considerable en el rendimiento del estudiante (Ammermueller, 2012; Ponzo, 2013; FrugårdStrøma, Thoresena, Wentzel-Larsena, \&Dyb, 2013). Existe una incidencia muy negativa sobre el rendimiento escolar de aquellos estudiantes que sufren de actos de violencia o intimidación, pero esta situación del entorno escolar también afecta los otros estudiantes, razón por la cual, el rol del profesor y de los pares resulta relevante, pues su acción de apoyo y ayuda puede atenuar en parte esos efectos negativos (Schenke, Lam, Conley, \&Karabnick, 2015). Aumentar el rendimiento académico entre los 
niños víctimas de maltrato, puede contribuir al desarrollo de sus habilidades para la vida diaria, y protegerlos de situaciones crónicas de maltrato (Coohey, Renner, Hua, Zhang, \& Whtney, 2011).

La investigación que se presenta, asume una perspectiva global de la convivencia escolar, pues busca visualizar e identificar el perfil del quehacer interno de la comunidad escolar, desde la perspectiva de los estudiantes. Con ello se determina la percepción de éstos acerca de aspectos institucionales como la gestión interpersonal positiva y la implicación del profesorado, pero al mismo tiempo, incorpora la perspectiva de los mismos jóvenes acerca de las situaciones que experimenta al interior del aula, o situaciones que pueden afectar su aprendizaje, como la indisciplina o disruptividad, su forma de atender, acoger o respetar, la reglamentación o la normativa interna, como también, y de forma relevante, la eventual ocurrencia de situaciones de agresión o acoso escolar que viva o de la cual sea testigo el propio estudiante. A partir de este constructo global, se persigue establecer el grado de relación entre la percepción de los estudiantes acerca de la convivencia en sus múltiples dimensiones y el rendimiento académico observado en matemática.

El presente artículo tiene por objetivo caracterizar a los estudiantes que obtienen buen rendimiento en la asignatura de Matemática en función a su percepción de la convivencia escolar en el aula y en su establecimiento. Las preguntas que guiaron esta investigación fueron: ¿Qué dimensiones de la convivencia escolar tienen mayor influencia en el rendimiento en Matemática? ¿Los niños y niñas con buen rendimiento que estudian en establecimientos municipales (públicos) tienen una percepción acerca de la convivencia escolar similar a los de colegios particulares subvencionados (concertados)? ¿La percepción de la convivencia escolar impacta sobre el rendimiento en Matemática de la misma forma en niños que en niñas?

\section{Método}

\section{Participantes}

En el estudio participaron 314 niños y niñas de educación primaria chilena que cursaban quinto año de educación primaria en establecimientos educacionales municipalizados y particulares subvencionados (ver Tabla 1) con una edad promedio de 10.46 años (ds $=0.51$ ).

Tabla 1.Distribución de estudiantes de la muestra por sexo y dependencia

\begin{tabular}{lllll} 
& Hombre & \multicolumn{3}{c}{ Mujer } \\
\hline $\begin{array}{l}\text { Establecimiento de dependencia Municipal } \\
\begin{array}{l}\text { Establecimiento de dependencia Particular } \\
\text { subvencionada }\end{array}\end{array}$ & 64 & 20.4 & 66 & 21.0 \\
\hline
\end{tabular}

\section{Instrumento}

La Escala de Convivencia Escolar (E.C.E.), es un instrumento que consta de 50 ítems agrupados en ocho dimensiones validadas estadísticamente por Del Rey et al. (Del Rey, Casas, \& Ortega-Ruiz, 2017) para caracterizar la percepción de la convivencia escolar de niños y niñas 
de enseñanza básica. La escala examina la percepción de los estudiantes en torno a tres tópicos: situaciones que se presentan en su colegio y su curso a nivel general y particular; cantidad de veces que ocurren situaciones que afectan a la convivencia en su establecimiento; y por último, si ha sido partícipe de determinados eventos y con qué frecuencia lo hace. Las dimensiones examinadas por la escala son: Gestión interpersonal positiva, Victimización, Disruptividad, Red Social de Iguales, Agresión, Ajuste Normativo, Indisciplina y Desidia docente. Todas las aseveraciones o rasgos que componen la escala son del tipo escala Likert, pues los estudiantes deben manifestar su nivel de acuerdo o desacuerdo respecto de ellas y están graduadas desde con una graduación que va desde $0=$ Nunca a $4=$ Siempre.

La validación de la escala realizada por los autores originales para la población escolar española, permitió identificar una solución factorial que consta de ocho dimensiones que explican el 63\% de la variabilidad total con un alfa de Cronbach de 0.75 . El puntaje obtenido en cada uno de los factores muestra la percepción del estudiante respecto a su comportamiento y el reflejado en su establecimiento.

En el estudio de adaptación a la población escolar Chilena, el análisis factorial confirmó y validó el modelo de 8 factores y presentó índices de ajuste muy adecuados: $\chi 2 \mathrm{SB}=2708.8, \mathrm{p}=$ .0001 ; CFI $=.969$; NNFI= .966; RMSEA= .035; IC (0.003-0.036), con un alfa de Cronbach de 0.80 (Cerda, \& Pérez, 2015).

Para determinar el rendimiento académico de los estudiantes en matemática, se recogieron sus promedios de calificaciones, que se registran en una escala de valores de 1 a 7 . Las dimensiones de la escala de convivencia escolar, el género y el tipo de dependencia administrativa del establecimiento del cual proviene el estudiante fueron consideradas como variables independientes; y las dos últimas, como variables dummy en el modelo.

\section{Procedimiento}

Inicialmente se realizó un análisis descriptivo de la ECE mediante el cálculo de estadígrafos. Posteriormente se construyeron modelos de regresión múltiple, ordinal y logística, categorizando la variable dependiente en los dos últimos casos. En la regresión ordinal, el procedimiento de categorización de la variable de respuesta se realizó de forma arbitraria, definiendo tres grupos: buen rendimiento (6.0 a 7.0), rendimiento regular (5.0 a 5.9) y mal rendimiento (igual o inferior a 4.9). Para la regresión logística se definió el evento "obtener buen rendimiento en Matemática” dicotomizando la variable de respuesta en las categorías 1 (el hecho ocurre) y 0 (el hecho no ocurre).

\section{Resultados}

El análisis descriptivo se realizó en Excel 2016® calculando estadígrafos de las ocho dimensiones del cuestionario de convivencia escolar para evaluar la percepción de la muestra en relación al constructo que mide el instrumento. Como se aprecia en la Tabla 2, existe una gran heterogeneidad en Agresión, la cual viene reflejada por el valor superior al $100 \%$ que exhibe el coeficiente de variación (cv), que expresa la relación entre la desviación típica y la media. Asimismo, las dimensiones de Victimización e Indisciplina también exhiben alta heterogeneidad, mientras que las dimensiones de Gestión Interpersonal Positiva y Ajuste 
Normativo presentan una alta homogeneidad, caracterizándose la muestra por una media elevada en estas dos últimas dimensiones respecto a su puntaje máximo.

Tabla 2. Estadígrafos de las ocho dimensiones de la escala convivencia escolar.

\begin{tabular}{lccccccc}
\hline Dimensión & $\begin{array}{c}\mathrm{N}^{\circ} \\
\text { ítems }\end{array}$ & $\begin{array}{c}\text { Puntaje } \\
\text { máximo }\end{array}$ & $\begin{array}{c}\text { Máximo } \\
\text { observado }\end{array}$ & $\begin{array}{c}\text { Mínimo } \\
\text { observado }\end{array}$ & $\overline{\boldsymbol{x}}$ & $\boldsymbol{s}$ & $\boldsymbol{c v}$ \\
\hline $\begin{array}{l}\text { Gestión Interpersonal Positiva } \\
\text { (GIP) }\end{array}$ & 11 & 44 & 44 & 10 & 37.4 & 5.6 & $15 \%$ \\
Victimización & 6 & 24 & 24 & 0 & 4.8 & 4.3 & $90 \%$ \\
Disruptividad & 6 & 24 & 23 & 1 & 10.8 & 4.9 & $45 \%$ \\
Red Social de Iguales (RSI) & 9 & 36 & 36 & 8 & 26.9 & 5.9 & $22 \%$ \\
Agresión & 4 & 16 & 16 & 0 & 2.7 & 2.9 & $110 \%$ \\
Ajuste Normativo & 5 & 20 & 20 & 0 & 16.1 & 3.7 & $23 \%$ \\
Indisciplina & 4 & 16 & 16 & 0 & 4.8 & 3.5 & $73 \%$ \\
Desidia Docente & 5 & 20 & 20 & 0 & 7.0 & 4.2 & $60 \%$ \\
\hline
\end{tabular}

Posteriormente y para una mejor representación, se reescalaron los puntajes; dividiendo el puntaje original por el número de variables que componen la dimensión y se desglosó la información por género y tipo de dependencia administrativa. Estos resultados son presentados gráficamente en la Figura 1.

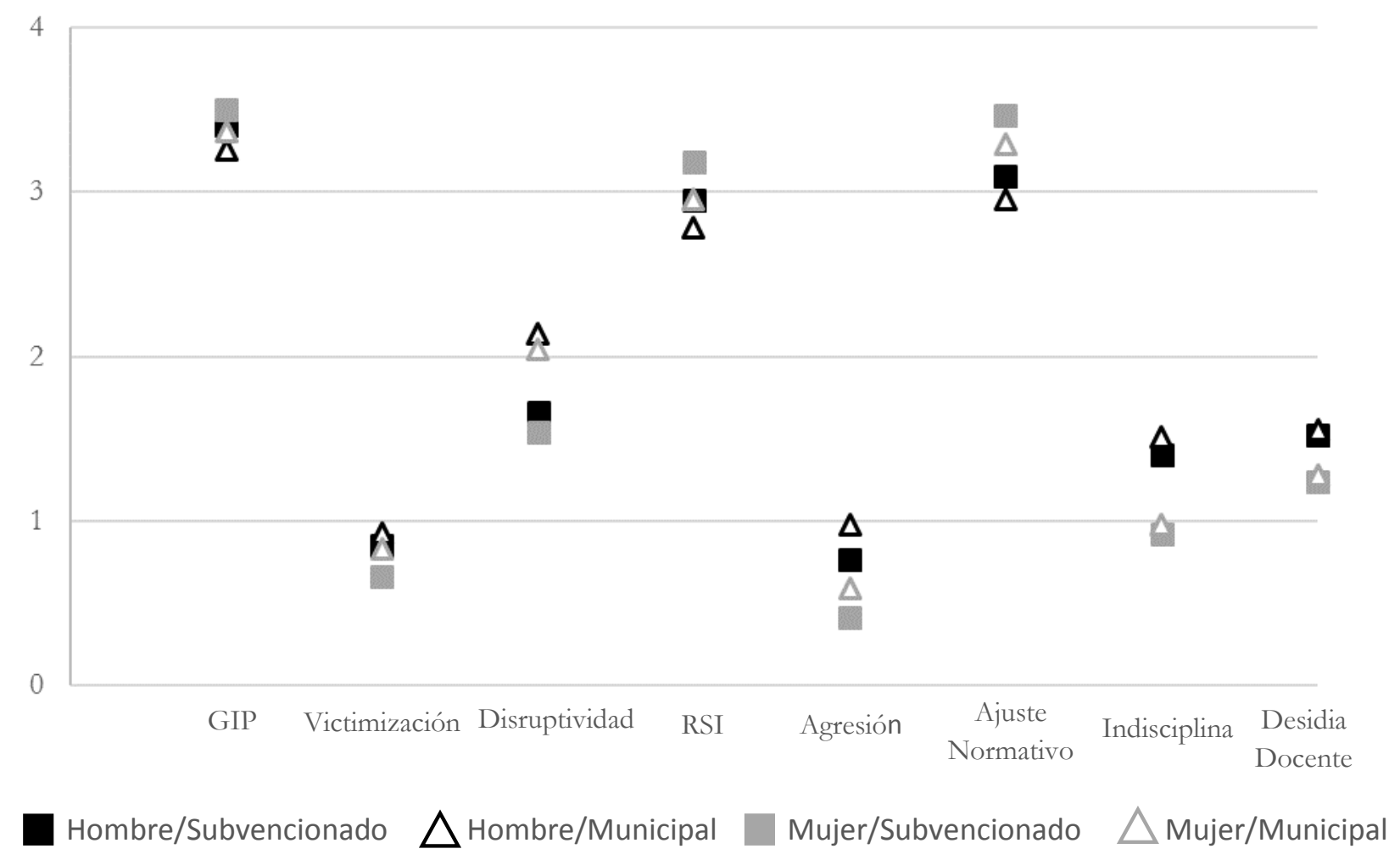

Figura 1. Representación gráfica de los puntajes reescalados por sexo y tipo de dependencia administrativa. 
De la Figura 1, se observa que las diferencias más importantes ocurren en las dimensiones de Disruptividad, Indisciplina y Desidia Docente. En la primera, se aprecian diferencias por tipo de dependencia administrativa, pero no por sexo. En cambio, en el caso de las dimensiones de Indisciplina y Desidia Docente, se visualiza una diferencia por sexo, pero no por tipo de dependencia administrativa. Por otro lado, en la dimensión Red Social de Iguales, se observa una clara diferencia entre niños de establecimientos municipales y niñas de colegios subvencionados, en donde estas últimas obtienen en promedio mayores puntuaciones.

Para determinar los perfiles de alumnos con buen rendimiento en Matemática en función a su percepción de la convivencia escolar, se consideraron tres tipos de regresión; lineal múltiple, ordinal y logística. La primera se descartó al constatar que el modelo explicaba sólo el 17,3\% de la variabilidad del rendimiento, y pronosticaba valores mayores al máximo posible (la calificación máxima equivale a 7.0). Para evitar esto, se categorizó la variable dependiente en tres niveles definiendo los grupos; buen rendimiento (6.0 a 7.0), rendimiento regular (5.0 a 5.9), mal rendimiento (igual o inferior a 4.9). Posteriormente, se realizó una regresión ordinal obteniendo un R cuadrado de Nagelkerke de 0.18. Dicho modelo tampoco logró clasificar de manera adecuada, ya que acertó alrededor de un 50\% en niños y niñas con buen rendimiento y rendimiento regular en Matemática, pero tendía a confundir un mal rendimiento académico con un rendimiento regular. Probablemente, estos dos grupos tengan las mismas percepciones sobre la convivencia escolar, por lo que se utilizó una regresión logística para considerar dos categorías en la variable de respuesta y evaluar la eficacia del modelo. Se consideraron, al igual que en las dos regresiones anteriores, las dimensiones de la escala de convivencia escolar, el sexo (1: Mujer, 0: Hombre) y el tipo de dependencia administrativa del establecimiento (1: Subvencionado, 0: Municipal) como variables independientes.

Debido a la naturaleza del algoritmo utilizado en el programa SPSS® se consideraron en el modelo de regresión logística, interacciones de primer orden y se utilizó la Curva ROC (Receiver OperatorsCharacteristic) para identificar el mejor punto de corte considerando como variables, la probabilidad predicha y la observada. El valor óptimo, donde la sensibilidad y especificidad se maximizaron (0.682 y 0.696, respectivamente) fue 0.36 . El modelo, sin posibles puntos influenciales (distancias de Cook menores que 1) explicó según el R cuadrado de Nagelkerke un 17,5\% y clasificó adecuadamente a ambas categorías (sobre 67\% de acierto). Los valores resultantes de los parámetros del modelo son presentados en la Tabla 3.

Tabla 3. Estimación de parámetros del modelo de regresión logística

\begin{tabular}{lccccc}
\hline Parámetro & $\boldsymbol{\beta}$ & Error estándar & Wald & gl & $\operatorname{Exp}(\boldsymbol{\beta})$ \\
\hline Constante & 0.20 & 0.35 & 0.35 & 1 & 1.23 \\
\hline Agresión & -0.11 & 0.07 & 2.32 & 1 & 0.90 \\
\hline Indisciplina & $-0.13 *$ & 0.06 & 4.51 & 1 & 0.88 \\
\hline Sexo (1) & 0.34 & 0.45 & 0.58 & 1 & 1.40 \\
\hline Agresión*Sexo(1) & $0.25 *$ & 0.11 & 5.17 & 1 & 1.29 \\
\hline Indisciplina*Sexo (1) & $0.24 *$ & 0.10 & 5.64 & 1 & 0.79
\end{tabular}

Nota. ${ }^{*} \mathrm{p}<.05, * * \mathrm{p}<.005, * * * \mathrm{p}<.001$ 
De la Tabla 3, se observa que las variables de Agresión e Indisciplina presentan de interacción con el sexo. En consecuencia, se generaron dos modelos que fueron representados gráficamente para una mejor interpretación (ver Figura 2). Se aprecia que, a mayor puntuación en Indisciplina, menor es la probabilidad que se obtenga un buen rendimiento, y también que hay mayor probabilidad de obtener un buen rendimiento en Matemática con una percepción baja tanto en Agresión como en Indisciplina. Además, se aprecia que la curva es más pronunciada en el caso de las niñas que para los niños, por lo que el impacto de la percepción de la Agresión e Indisciplina sobre el rendimiento resulta menor en hombres que en mujeres.

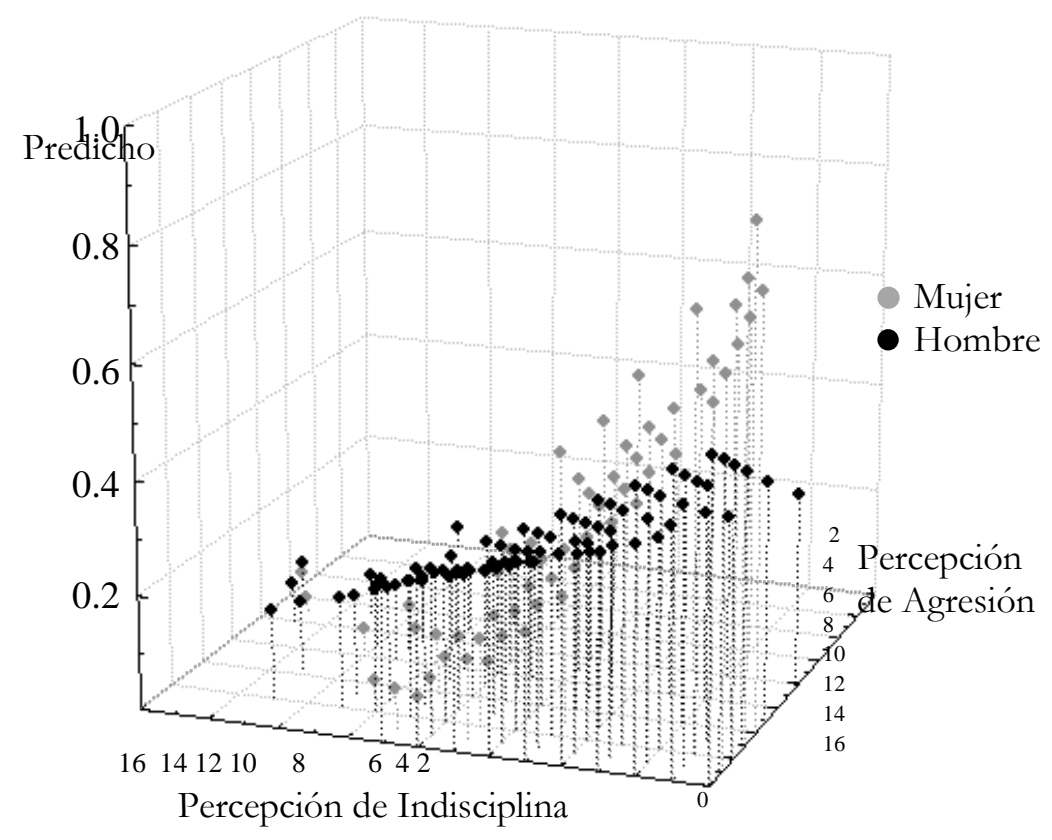

Figura 2. Representación gráfica de los dos modelos de rendimiento en Matemática obtenidos por regresión logística.

\section{Discusión}

Los resultados de la investigación dan cuenta de una relación estrecha e importante entre la percepción de las diversas dimensiones de la convivencia escolar que tienen los estudiantes en sus respectivos establecimientos escolares y el rendimiento académico en matemática que ellos presentan.

Se destaca el rol negativo que tienen las dimensiones de agresión e indisciplina y su interacción con el género de los estudiantes, sumado a la dependencia administrativa de los establecimientos escolares a los cuales asisten los estudiantes. El efecto de interacción de dichas variables sobre el rendimiento en matemáticas es más acusado entre las chicas, que en el grupo de los chicos.

Así, es posible observar que aquellos estudiantes que tienden a percibir un mayor nivel de involucramiento en actos agresivos hacia sus pares, realizando actos como descalificaciones, insultos o golpes, tienden a tener un peor desempeño en matemática. Es probable que para el 
grupo de mujeres, las situaciones de indisciplina o agresividad que surgen al interior del establecimiento afecten en mayor medida su desempeño en el área de matemática. Existen varias conjeturas que se pueden aportar para explicar este comportamiento diferencial. Por una parte, se constata que los chicos agreden más que las chicas (Ortega y Mora-Merchán, 2008) y que las chicas perciben menores expectativas de parte de sus profesores con respecto a sus habilidades en matemática (Lazarides, \& Watt, 2015). Numerosas investigaciones dejan en evidencia, además, que la actitud negativa hacia el aprendizaje de la matemática es más acusada entre las chicas, y presentan mayores niveles de ansiedad frente a las tareas en éste ámbito, y menores niveles de autoeficacia (Akin, \&Kurbanoglu, 2011; Selkirk, Bouchey, \&Eccles, 2011). Es posible afirmar que podría haber patrones emocionales en las mujeres basados en factores culturales como creencias y estereotipos de menor dominio en matemáticas y, por ende, más baja competencia en el área (Nosek, \&Smyth, 2011). En cuanto a los niños, los resultados no son concluyentes (Belfi, Goos, De Fraine, \& Van Damme, 2012).

Del mismo modo, los estudiantes que perciben un mayor nivel de actos de indisciplina escolar al interior de su establecimiento, tienden a mostrar peores promedios de calificaciones en matemática que aquellos de sus iguales que tienen una percepción más favorable en esos aspectos de la convivencia escolar. Probablemente, las situaciones de indisciplina escolar afecten el grado de aprovechamiento de los objetivos de aprendizaje en dicha disciplina escolar, pues estas situaciones no sólo afectan la atención requerida para el aprendizaje, o el establecimiento de un ambiente propicio para que el aprendizaje ocurra, sino que también afectan en muchos casos la motivación de los estudiantes (Arens, Morin, \&Watermann, 2015). Se suma a esta condición desfavorable, el hecho que en las escuelas públicas chilenas a las que asiste la población socioeconómicamente más vulnerable, se ha constatado que los estudiantes se sienten más estigmatizados y discriminados por variables académicas (Ascorra, López, \& Núñez, 2016) .

Por otra parte, se ha encontrado una correlación negativa entre la percepción de victimización y el rendimiento escolar en matemáticas. Es decir, aquellos estudiantes que se sienten insultados, agredidos o afectados por cualquier situación de acoso o violencia escolar, exhiben promedios de calificaciones más bajos en matemáticas. Estos hallazgos son coincidentes con investigaciones que demuestran que ser víctima de acoso o intimidación escolar tiene un efecto negativo considerable en el rendimiento del estudiante (Ammermueller, 2012; Ponzo, 2013).

Estos hallazgos permiten relevar el rol de los agentes educativos, especialmente de la gestión del docente al interior del aula escolar, pues si se interviene o propicia una influencia positiva entre los pares que permita una atmósfera o clima de clase adecuado para el desarrollo del proceso enseñanza aprendizaje, todo ello ejerce un efecto indirecto y positivo en el rendimiento en matemáticas. En otras palabras, la responsabilidad del profesor respecto de la elección del enfoque didáctico, la metodología, recursos o actividades, debe tener como meta transversal y permanente el favorecer un espacio propicio para el aprendizaje, flexible y desafiante, que facilite el surgimiento de relaciones dialógicas de mutuo respeto.

Los resultados sugieren que el establecer relaciones positivas y simétricas con los pares y profesores, puede actuar como un elemento catalizador de conductas agresivas, al permitir manejar la ira y demorar la búsqueda de la urgente necesidad de gratificación de los estudiantes, la que generalmente se asocia a indisciplina y acoso escolar, por lo cual 
colateralmente se morigera este comportamiento disruptivo favoreciendo el aprendizaje y rendimiento académico (Herndon, \&Bembenutty, 2014). Adicionalmente, existe evidencia que muestra que el compromiso de enseñanza por parte de los profesores, expresado en acciones específicas tales como fomentar la participación en la clase y corregir las tareas de los estudiantes, se relaciona positivamente con un mejor desempeño de éstos en la asignatura de matemáticas. Esta supervisión activa por parte de los profesores, relacionada con las dimensiones de gestión interpersonal positiva y desidia docente del constructo convivencia escolar examinado, propicia adicionalmente, buenas relaciones interpersonales con los padres, lo que a su vez, tiene un efecto positivo en el rendimiento (Kodzi, et al., 2014).

Por otra parte, existe una incidencia muy negativa sobre el rendimiento escolar en aquellos estudiantes que sufren de actos de violencia o intimidación, pero esta situación del entorno escolar también afecta a los otros estudiantes, razón por la cual el rol del profesor y de los pares resulta relevante, pues su acción de apoyo y ayuda puede atenuar en parte esos efectos negativos (Schenke, et al., 2015). Aumentar el rendimiento académico entre los niños víctimas de maltrato, contribuye al desarrollo de sus habilidades para la vida diaria (Coohey et al., 2011).

Adicionalmente, los profesores pueden desempeñar un rol relevante en la promoción de búsqueda de ayuda y perseverancia por parte de los estudiantes al enfrentar la actividad matemática. Al enfatizar las metas de dominio y hacer la tarea más interesante, esto permite que los estudiantes puedan aprender y sostener la motivación por la tarea, del mismo modo que alientan a las familias de éstos a estar disponibles para apoyar dicho proceso $(\mathrm{Du}, \mathrm{Xu}, \& \mathrm{Fan}$, 2016).

Del mismo modo, la investigación muestra que los estudiantes que asisten a colegios que se sitúan en contextos de mayor vulnerabilidad social, y al cual asisten estudiantes de menores ingresos económicos como los públicos o de dependencia administrativa municipal, tienen más bajas calificaciones que sus iguales que asisten a colegios concertados o de dependencia administrativa Particular Subvencionada. Esto es coincidente con el alto grado de estratificación social del sistema escolar chileno, hecho relevado en numerosas investigaciones realizadas en el contexto de la educación chilena, que da cuenta de la existencia de diferencias significativas en los niveles de rendimiento escolar en función del grupo de extracción social (Furnham, Monsen, \&Ahmetoglu, 2009; Martín, Martínez-Arias, Marchesi, \& Pérez, 2008), que incluso condicionan su futuro itinerario escolar y laboral (Del Solar, 2013; Echeñique , \& Urzúa, 2013). Lo anterior, no sólo pone de manifiesto un sistema escolar altamente inequitativo, que le resta oportunidades de acceso a aprendizajes de mejor calidad o logros académicos en matemáticas, sino que además interactúa de forma desfavorable con la presencia de condiciones adversas o negativas en las dimensiones de la convivencia señaladas en el estudio, que agravan su condición de entrada.

Finalmente, tal como ha quedado de manifiesto, los estudiantes en contextos vulnerables, presentan no sólo un desarrollo insuficiente de sus habilidades matemáticas, sino que además, esa condicionalidad se hace más desfavorable si se suma a dicho contexto escolar, el hecho que la convivencia escolar de su establecimiento presente problemas de indisciplina, agresividad y situaciones de acoso.

Finalmente, y en otro plano de cosas, las instituciones escolares chilenas parecen haber migrado a estar particularmente atentas a detectar y cuantificar los comportamientos violentos, 
el cumplimiento del o de los eventos que pueden contravenir las normas y reglamentos, con la finalidad de cumplir a cabalidad su plan de convivencia escolar y evitar de este modo las sanciones que la propia Ley de convivencia estipula. Los resultados de este estudio aportan otra dimensión que puede revestir de mayor interés aun la preocupación por mantener o fomentar un clima adecuado.

La escala ECE, se erige entonces como una herramienta muy adecuada para realizar un diagnóstico certero acerca de las diversas dimensiones de la convivencia escolar, permitiendo que las autoridades de las instituciones escolares asuman un examen crítico, abierto y reflexivo sobre la propia responsabilidad de sus miembros y estamentos de la misma, evitando burocratizar estas acciones sólo al plano administrativo por sobre el compromiso cabal de crear una comunidad de convivencia (Magendzo, Toledo, \& Gutiérrez, 2013).

\section{Agradecimientos}

Trabajo apoyado por el proyecto Fondecyt de Iniciación N 11150201. "Logros en matemática: modelando la compleja interacción de factores de la convivencia escolar, disposición emocional, disponibilidad léxica matemática y niveles de inteligencia lógica en establecimientos de alta vulnerabilidad”, el Proyecto Basal FB0003 del Programa de Investigación Asociativa de CONICYT, y el Proyecto EDPG-LPR-164, Dirección de Postgrado, Universidad de Concepción.

\section{Referencias}

Agencia de Calidad de la Educación. (2014). Informe Técnico SIMCE 2013. Santiago, Gobierno de Chile.

Akin, A., \&Kurbanoglu, I. (2011). The relationships between math anxiety, math attitudes, and self- efficacy: A structural equation model. Studia Psychologica, 53(3), 263-274.

Ammermueller, A. (2012). Violence in European schools: A widespread phenomenon that matters for educational production. Labour Economics 19, 908-922. V. doi.org/10.1016/j.labeco.2012.08.010

Arens, K., Morin, A., \& Watermann, R. (2015). Relations between classroom disciplinary problems and student motivation: Achievement as a potential mediator? A Learning and Instruction 39, 184-193. doi.org/10.1016/j.learninstruc.2015.07.001.

Arístegui, R., Bazán, D., Leiva, J., Muñoz, B., y Ruiz, J. (2005). Hacia una pedagogía de la convivencia. Psykhe, 14(1), 137-150. DOI:10.4067/S0718-22282005000100011

Ascorra, P., López, V., Núñez, C. G., Bilbao, M. A., Gómez, G., \& Morales, M. (2016). Relación entre segregación y convivencia escolar en escuelas chilenas con altos puntajes PISA 2009. Universitas Psychologica, 15(1), 65-78. doi.org/10.11144/Javeriana.upsy151.rsce

Bandalos, D. L., Finney, S. J. \&Geske, J. A. (2003). A model of statistics performance based on achievement goal theory. Journal of Educational Psychology, 95(3), 604-616. DOI: 10.1037/0022-0663.95.3.604. 
Belfi, B., Goos, M., De Fraine, B., \& Van Damme, J. (2012). The effect of class composition by gender and ability on secondary school students' school well-being and academic self-concept: A literature review. Educational Research Review 7, 62-74. doi:10.1016/j.edurev.2011.09.002

Brand, S., Felner, R., Seitsinger, A., Burns, A. \& Bolton, N. (2008). A large scale study of the assessment of the social environment of middle and secondary schools: The validity and utility of teachers' ratings of school climate, cultural pluralism, and safety problems for understanding school effects and school improvement. Journal of School Psychology, 46 (5), 507-535. doi:10.1016/j.jsp.2007.12.001

Brault, M.C., Janosz, M., \&Archambaul, M. (2014). Effects of school composition and school climate on teacher expectations of students: A multilevel analysis. Teaching and Teacher Education 44, 148-159. doi.org/10.1016/j.tate.2014.08.008

Cohen, J,. McCabe, L, Michelli, N.M \&Pickeral, T. (2009). School Climate: Research, Policy, Teacher Education and Practice. Teachers College Record, 111 (1), 180-213.

Coohey,C., Renner, L., Hua, L., Zhang, Y., \&Whtney, S. (2011). Academic achievement despite child maltreatment: A longitudinal study. Child Abuse \& Neglect 35, 688-699. doi:10.1016/j.chiabu.2011.05.009

De Pedro, K.T., Gilreath, T., \& Berkowitz, R. (2016). A latent class analysis of school climate among middle and high school students in California public schools. Children and Youth Services Review, 63, 10-15. doi.org/10.1016/j.childyouth.2016.01.023

Del Rey, R., Casas, J. A., \& Ortega Ruiz, R. (2017). Desarrollo y validación de la Escala de Convivencia Escolar (ECE). Universitas Psychologica, 16(1), xx-Xx. doi.org/10.11144//Javeriana.upsy16-1.dvec

Del Solar, B. (2013). Combatiendo la desigualdad. Puntos de Referencia. Centros de Estudios Públicos, 364.

Djigic, G. \&Stojiljkovic, S. (2011). Classroom management styles, classroom climate and school achievement. Procedia Social and Behavioral Sciences, 29, 819-828. doi:10.1016/j.sbspro.2011.11.310

Doris, A., O’Neill, D., \& Sweetman, O. (2013). Gender, single-sex schooling and maths achievement. Economics of Education Review 35, 104-119. doi.org/10.1016/j.econedurev.2013.04.001

Du, J., Xu, J. \& Fan, X. (2016) Investigating factors that influence students' help seeking in math homework: A multilevel analysis. Learning and Individual Differences, 48, 29-35. doi.org/10.1016/j.lindif.2016.03.002

Echeñique, J. y Urzúa, S. (2013). Desigualdad, Segregación y Resultados Educacionales. Evidencia desde el Metro de Santiago. Puntos de Referencia. Centro de Estudios Públicos. http://www.cepchile.cl/dms/archivo_5287_3385/pder359_SUrzuaJEchenique.pdf

FrugårdStrøma, I., Thoresena, S., Wentzel-Larsena, T. \&Dyb, G. (2013). Violence, bullying and academic achievement: A study of 15-year-old adolescents and their school environment. Child Abuse \& Neglect, 37 (2013) 243-251. doi.org/10.1016/j.chiabu.2012.10.010

Furnham, A., Monsen, J. y Ahmetoglu, G. (2009). Typical intellectual en-gagement, Big Five personality traits, approaches to learning and cogni-tive ability predictors of academic 
performance. British Journal of Educational Psychology, 79(4), 769-782.

Gázquez, J.J., Pérez, M.C., \& Carrión, J.J. (2011).Clima escolar y resolución de conflictos según el alumnado: un estudio europeo. Revista de Psicodidáctica, 16(1), 39-58.

González-Castro, P., Álvarez-García, D., Rodríguez, C., Álvarez, L., \& Núñez, J. C. (2010). La formación de los futuros docentes frente a la violencia escolar. Revista de Psicodidáctica, 15(1), 35-56.

Henrich, C., Schwab-Stone, M., Fanti, K., Jones, S. \&Ruchkin, V. (2004). The association of community violence exposure with middle-school achievement: A prospective study. Applied Developmental Psychology, 25, 327-348. doi:10.1016/j.appdev.2004.04.004

Herndon, J.S. \&Bembenutty, H. (2014). In-school and social factors influencing learning among students enrolled in a disciplinary alternative school. Learning and Individual Differences, 35, 49-55. doi.org/10.1016/j.lindif.2014.07.007

Hopson, L.M. \& Lee, E. (2011) Mitigating the effect of family poverty on academic and behavioral outcomes: The role of school climate in middle and high school. Children and Youth Services Review, 33, 2221-2229. doi:10.1016/j.childyouth.2011.07.006

Kaynak, Ö.,Lepore, S., Kliewer, W., \& Jaggi, L.,(2015). Peer victimization and subsequent disruptive behavior in school: The protective functions of anger regulation coping. Personality and Individual Differences, 73, 1-6. doi.org/10.1016/j.paid.2014.09.012

Kodzi, I., Oketch, M., Ngware, M., Mutisya, M., \&Nderu, E. (2014). Social relations as predictors of achievement in math in Kenyan primary schools. International Journal of Educational Development, 39, 285-292. doi.org/10.1016/j.ijedudev.2014.02.007

Lazarides, R., \& Watt, H. (2015). Girls' and boys' perceived mathematics teacher beliefs, classroom learning environments and mathematical career intentions. Contemporary Educational Psychology, 41, 51-61. doi:10.1016/j.cedpsych.2014.11.005

Ley 20.536 (2011). Ley sobre Violencia Escolar . Ministerio de Educación. Gobierno de Chile. Retrieved from https://www.leychile.cl/Navegar?idNorma=1030087

Magendzo K, Abraham, Toledo J, María I, \& Gutiérrez G, Virna. (2013). Descripción y análisis de la Ley sobre Violencia Escolar ( $\left.\mathrm{N}^{\circ} 20.536\right)$ : dos paradigmas antagónicos. Estudios pedagógicos (Valdivia), 39(1), 377-391. doi.org/10.4067/S071807052013000100022

Martín, E., Martínez-Arias, R., Marchesi, A., \& Pérez E. (2008). Variables that predict academic achievement in the Spanish compulsory Secondary Educational System: A longitudinal multi-level analysis. The Spanish Journal of Psychology, 11(2), 400-413.

Martorell, P., Stange, K., \&McFarlin, I. (2016). Investing in schools: capital spending, facility conditions, and student achievement. Journal of Public Economics, 140, 13-29. http://dx.doi.org/10.1016/j.jpubeco.2016.05.002

Meristo, M. \&Eisenschmidt, E. (2014). Novice teachers' perceptions of school climate and self-efficacyInternational Journal of Educational Research 67, 1-10. doi.org/10.1016/j.ijer.2014.04.003

MINEDUC (2010a). Resultados Nacionales SIMCE. Ministerio de Educación, Unidad de Curriculum y Evaluación.

MINEDUC (2010b). Resumen de Resultados PISA 2009 Chile. Ministerio de Educación, Unidad de Curriculum y Evaluación. 
MINEDUC (2014). Resultados TIMSS 2011 Chile. Estudio Internacional de Tendencias en Matemática. Ministerio de Educación, Agencia de Calidad de la Educación División de Estudios. Disponible

en www.agenciaeducacion.clCienciashttp://portales.mineduc.cl/usuarios/acalidad/doc/2013 01151653440.Informe_Resultados_TIMSS_2011_Chile_(10-01-13).pdf

Miñano, P., \& Castejón, J. L. (2011). Variables cognitivas y motivacionales en el rendimiento académico en Lengua y Matemáticas: un modelo estructural. Revista de Psicodidáctica, 16(2), 203-230.

Mitchell, M., \& Bradshaw, C., (2013). Examining classroom influences on student perceptions of school climate: The role of classroom management and exclusionary discipline strategies. Journal of School Psychology, 51, 599-610. doi.org/10.1016/j.jsp.2013.05.005

Nakamoto, J., \& Schwartz, D. (2011). The association between peer victimization and functioning at school among urban Latino children. Journal of Applied Developmental Psychology, 32, 89-97. doi:10.1016/j.appdev.2011.02.003

Nosek, B. A. \& Smyth, F. L. (2011). Implicit social cognitions predict sex differences in math engagement and achievement. American Educational Research Journal, 48, 1124-1154. DOI: $10.3102 / 0002831211410683$

Ortega, R., y Mora-Merchán, J. (2008). Las redes de iguales y el fenómeno del acoso escolar: Explorando el esquema dominio-sumisión. Infancia y Aprendizaje, 31(4), 515-528

Perkins, S. \& Graham-Bermann, S. (2012). Violence exposure and the development of schoolrelated functioning: Mental health, neurocognition, and learning. Aggression and Violent Behavior, 17, 89-98. doi:10.1016/j.avb.2011.10.001

Ponzo, M. (2013). Does bullying reduce educational achievement? An evaluation using matching estimators. Journal of Policy Modeling, 35, 1057-1078. doi.org/10.1016/j.jpolmod.2013.06.002

Sánchez Moreno, M. \&LópezYáñez, J. (2010). Culturas institucionales que facilitan y dificultan la mejora de la escuela. Profesorado. Revista de Curriculum y Formación del Profesorado, 14(1), 93-110.

Schenke, K., Lam, A.M., Conley, A. \& Karabnick, S. (2015). Adolescents' help seeking in mathematics classrooms: Relations between achievement and perceived classroom environmental influences over one school year. Contemporary Educational Psychology 41, 133-146. doi:10.1016/j.cedpsych.2015.01.003

Selkirk, L., Bouchey, H., \& Eccles, J. (2011). Interactions among domain-specific expectancies, values, and gender: Predictors of test anxiety during early adolescence. Journal of Early Adolescence, 31(3), 361-389. DOI: 10.1177/0272431610363156

Tavares, P.A. (2015). The impact of school management practices on educational performance: Evidence from public schools in São Paulo. Economics of Education Review, 48, 1-15. doi.org/10.1016/j.econedurev.2015.05.002

Toledo, M. I., Magendzo, A. y Gutiérrez, V. (2009). Relación entre intimidación (bullying) y clima en la sala de clases y su influenza sobre el rendimiento de los estudiantes. Fondo de Investigación y Desarrollo en Educación. Chile: Mineduc. 
Tosto, M.G., Asbury, K., Mazzocco, M., Petrill, S., \& Kovas, Y. (2016) (in press). From classroom environment to mathematics achievement: The mediating role of selfperceived ability and subject interest. Learning and Individual Differences. doi.org/10.1016/j.lindif.2016.07.009

Zullig, K. J., Koopman, T. M., Patton, J. M., \& Ubbes, V. A. (2010). School climate: Historical review, instrument development, and school assessment. Journal of Psychoeducational Assessment, 28, 139 -152. Doi.org/10.1177/07342829093442 